TRANSACTIONS OF THE

AMERICAN MATHEMATICAL SOCIETY

Volume 192, Issue 6, 1974

\title{
GOLDIE-LIKE CONDITIONS ON JORDAN MATRIX RINGS(1)
}

BY

DANIEL J. BRITTEN

ABSTRACT. In this paper Goldie-like conditions are put on a Jordan matrix ring $J=H\left(R_{n}, \gamma_{a}\right)$ which are necessary and sufficient for $R$ to be a *-prime Goldie ring or a Cayley-Dickson ring. Existing theory is then used to obtain a Jordan ring of quotients for $J$.

I. Introduction. Our basic reference for this paper is [5]. The Coordinatization Theorem [5, p. 137] states that any Jordan algebra over a field of characteristic $\nRightarrow 2$ with three or more connected idempotents which sum to 1 is isomorphic to a Jordan matrix algebra $H\left(R_{n}, \gamma_{a}\right)$ of symmetric elements with respect to a canonical involution $\gamma_{a}$. It is with this and Goldie's theorem for semiprime rings [4, p. 270] in mind that we approach the problem of quotient rings of Jordan rings and obtain a quotient ring which is of the type given in the Second Structure Theorem [5, p. 179].

II. Preliminaries and statement of main result. Let $J$ be a Jordan ring whose multiplication is denoted by "." For each $a \in J$ there is the U-operator given by $U_{a}(b)=2 a \cdot(a \cdot b)-a^{\cdot 2} \cdot b . J$ is said to be prime provided that, if $A$ and $B$ are ideals of $J$ and $U_{A}(B)=\left\{U_{a}(b): a \in A, b \in B\right\}=0$, then $A=0$ or $B=0$ [8]. $Q \subseteq J$ is said to be a quadratic ideal provided $Q$ is an additive subgroup of $(J,+)$ and $U_{Q}(J) \subseteq Q$.

If we let $R$ be a nonassociative ring with characteristic $\neq 2$ such that $1 / 2 \in R$ and ${ }^{*}$ is an involution on $R$, then the set of symmetric elements under a canonical involution (see [5, p. 125]), $J=H\left(R_{n}, \gamma_{a}\right)$, is a Jordan ring for $n \geq 3$ with product $c \cdot b=1 / 2(c b+b c)$ if and only if either $R$ is associative or $n=3$ and $R$ is alternative with its set of symmetric elements $H$ contained in its nucleus $N$ [5, p. 127].

Let $a=\operatorname{diag}\left\{a_{1}, \ldots, a_{n}\right\}$ and the $a_{i}$ 's are symmetric invertible elements in the nucleus of $R$. The canonical involution $\gamma_{a}$ acts on the $n \times n$ matrix $X$ to give $a^{-1} X^{* t} a$ where $X^{* t}$ denotes the matrix obtained by applying ${ }^{*}$ to each of the entries of $X$ and then taking the transpose.

Received by the editors September 8, 1972.

AMS (MOS) subject classifications (1970). Primary 17A40; Secondary .16A58.

(1) Most of this paper appeared in the author's Ph.D. dissertation written under the direction of Professor F. Kosier at the University of Iowa. The author wishes to thank Professor Kosier for his help and encouragement. 
General assumption. In this paper: $\gamma_{a}$ is a canonical involution; $J=\left(R_{n}, \gamma_{a}\right)$, $n \geq 2$, is a Jordan ring; $1 / 2 \in R$ an alternative ring with involution * such that $H \subseteq N$.

If $z \in R$ and $\left\{e_{i j}\right\}$ is the standard set of matrix units, then we shall write $z_{i j}$ for $z e_{i j}+a_{j}^{-1} z^{*} a_{i} e_{j i}, i \neq j$, in $J$. Although we shall have occasion to talk about matrices over $R$ of more general form, we shall attempt to eliminate the confusion by reserving the subscripts $i$ and $j$ for this purpose, unless the matrix is written out. One can easily see that the elements of $J$ are merely sums of elements of the form $z_{i j}, i \neq j$, and elements of the form $b a_{i} e_{i i}$ where $b \in H$. We shall use $J_{i j}, i \neq j$, for $\left\{x_{i j}: x \in R\right\}$, and if $A \subseteq J$ then $A_{i j}=A \cap J_{i j}$ A quadratic ideal $Q$ is said to be an $i j$-quadratic ideal, $i \neq j$, provided $Q_{i j} \neq 0$.

The next two definitions are basic for our Goldie-like conditions.

Definition. A nonempty set $\left\{Q_{k}\right\}$ of distinct nonzero quadratic ideals will be called a direct system provided that, if $\left\{Q_{n}: n \in I_{1}\right\}$ and $\left\{Q_{l}: l \in I_{2}\right\}$ are finite subsets of $\left\{Q_{k}\right\}$, where $I_{1} \cap I_{2}=\varnothing$, then the quadratic ideal generated by $\Sigma Q_{n}$ intersected with the quadratic ideal generated by $\Sigma Q_{l}$ is 0 . The direct system is said to be infinite if $\left\{Q_{k}\right\}$ contains infinitely many quadratic ideals.

Definition. If $Q$ is a quadratic ideal in $J$, then the quadratic ideal $A$ generated by $\left\{z_{i j} \in J_{i j}: U_{z_{i j}}\left(Q_{i j}\right)=0\right\}$ is defined to be the $i j$-annihilators of $Q$, $i j$-ann $(Q)$, provided $U_{A_{i j}}\left(Q_{i j}\right)=0, i \neq j ;$ otherwise $i j$-ann $(Q)=0$.

Our Jordan analogue for the associative ring of quotients is given as follows:

Definition. The Jordan ring $J^{\prime}$ is said to be a Jordan ring of quotients for the Jordan ring $J$ provided:

(i) there exists an isomorphism $f: J \rightarrow J^{\prime}$ (we shall consider $J$ as a Jordan subring of $J^{\prime}$ );

(ii) every regular element $a$ in $J$ (i.e., $U_{a}$ is injective as it acts on $J$ ) is invertible in $J^{\prime}$;

(iii) every element of $J^{\prime}$ is of the form $U_{a}^{-1}(b)$ for $a, b$ in $J$ with $a$ regular in $J$.

Main Theorem. Let $R$ be an alternative ring with involution * sucb that $H \subseteq N$ and $1 / 2 \in R$ and let $J=H\left(R_{n}, \gamma_{a}\right), n \geq 2$, be a Jordan matrix ring. Then $J$ is prime, satisfies ACC on ij-annibilators, and contains no infinite direct system of ij-quadratic ideals if and only if either $R$ is a *-prime Goldie ring or $n=2,3$ and $R$ is a Cayley-Dickson ring. Moreover, in this case, the Jordan ring of quotients $J^{\prime}$ for $J$ is $J^{\prime}=H\left(R_{n}^{\prime}, \gamma_{a}\right)$ where $R^{\prime}$ is the ring of quotients for $R$ so that $R^{\prime}$ is a *-simple Artinian ring or a Cayley-Dickson algebra.

III. Only if. Throughout this section we shall assume that $J$ satisfies the conditions of the Main Theorem.

An ideal $A$ of $R$ is said to be a *-ideal if $A^{*}=A . R$ is said to be a *-prime provided that, if $A$ and $B$ are *-ideals and $A B=0$, then either $A=0$ or $B=0$. 
If $A$ is a *-ideal then $A \cap J$ is an ideal of $J$. Thus the primeness of $J$ gives the *-primeness of $R$ so that by [1] $R$ contains a prime ideal $P$ such that $P \cap P^{*}=0$ (we shall use the existence of this prime ideal throughout). Also by [1], this implies that $R$ is a Cayley-Dickson ring or $R$ is associative. Thus we assume that $R$ is associative, so that $U_{a}(b)=a b a$ for all $a, b \in \mathrm{J}$.

Theorem 3.1. If $J$ is prime and contains no infinite direct system of ij-quadratic ideals and $R$ is associative tben $R$ contains no infinite direct sum of one sided ideals.

Proof. Suppose $\Sigma L_{k}$ is an infinite direct sum of left ideals in $R$ and let $P$ be a prime ideal such that $P \cap P^{*}=0$. Then there are infinitely many $L_{k}$ 's not contained in $P$ or infinitely many $L_{k}$ 's not contained in $P^{*}$. We assume $L_{k} \not P$ for all $k_{0}$ Thus $\left(L_{k}^{*}+P^{*}\right)\left(L_{k}+P\right) \neq 0$ by the primeness of $P$ so that $B_{k}=$ $\left(L_{k}^{*}+P^{*}\right) \cap\left(L_{k}+P\right) \neq 0$. Let $0=\Sigma b_{k}$ such that $b_{k}=0$ for all but finitely many $k^{\prime}$ 's and $b_{k} \in B_{k}$, so that $b_{k}=l_{k}+p_{k}=l_{k}^{\prime *}+p_{k}^{\prime *}$ where $l_{k}, l_{k} \in L_{k}$ and $p_{k}, p_{k}^{\prime} \in P$. If $P=0$, then $b_{k}=0$ for all $k$ since $\Sigma L_{k}$ is a direct sum. If $P \neq 0$ then $0=$ $P^{*} 0=P^{*} \Sigma b_{k}$, so that $P^{*} l_{k}=0$. Thus $l_{k} \in P$ and hence $b_{k} \in P$. By a similar argument $b_{k} \in P^{*}$ so that in any case $b_{k}=0$. Therefore $\Sigma B_{k}$ is a direct sum of nonzero subgroups of $(R,+)$.

If we set $Q_{k}=\left\{b a_{i} e_{i i}+b a_{j} e_{i j}+b^{*} a_{i} e_{j i}+b^{\prime} a_{j} e_{j j}: b, b^{\prime} \in H \cap B_{k}\right.$ and $\left.b \in B_{k}\right\}$, it is easy to check that $Q_{k}$ is a quadratic ideal. Now let $\left\{Q_{n}\right\}_{n \in I_{1}}$ and $\left\{Q_{m}\right\}_{m \in I_{2}}$ be two finite disjoint subsets of $\left\{Q_{k}\right\}$. Let $Q_{l_{1}}$ be the quadratic ideal generated by $\Sigma_{n \in I_{1}} Q_{n}$ and $Q_{I_{2}}$ be the quadratic ideal generated by $\Sigma_{m \in I_{2}} Q_{m}$. Let $C=$ $\Sigma_{n \in I_{1}} L_{n}$ and $D=\Sigma_{m \in I_{2}} L_{m}$ so that $C+D$ is a direct sum of left ideals, and we may perform similar constructions using $C$ and $D$ to obtain $Q_{C}$ and $Q_{D}$, respectively, as we did to construct $Q_{k}$ using $L_{k}$. We then have $Q_{I_{1}} \subseteq Q_{C}$ and $Q_{I_{2}} \subseteq$ $Q_{D}$ and $Q_{I_{1}} \cap Q_{I_{2}} \subseteq Q_{C} \cap Q_{D}=0$ since

$$
\left[\left(C^{*}+P^{*}\right) \cap(C+P)\right] \cap\left[\left(D^{*}+P^{*}\right) \cap(D+P)\right]=0 .
$$

Thus we see that $\left\{Q_{k}\right\}$ is an infinite direct system of $i j$-quadratic ideals.

We now turn our attention to considering $i j$-annihilators. For a nonempty subset $S$ of $R$, we shall use $A_{L}(S), A_{R}(S)$ for the left, right annihilator of $S$, respectively. If $L=A_{L}(S)$ and $T=A_{R}(L)$, we see that $A_{L}(T)$ is $L$. We would like to use $T$ and $L$ to give some insight into $i j$-annihilators.

If $V$ is either a left or right ideal of $R$ then

$$
\begin{aligned}
Q_{j i}(V)=\left\{x e_{i i}+a_{i}^{-1} v^{*} a_{j} e_{i j}+v e_{j i}+y e_{j i}:\right. & \\
v & \left.\in V, x \in a_{i}^{-1}(V ! 1 H), \text { and } y \in H a_{j}\right\},
\end{aligned}
$$

and 


$$
\begin{aligned}
& Q_{i j}^{\prime}(V)=\left\{x e_{i i}+v e_{i j}+a_{j}^{-1} v^{*} a_{i} e_{j i}+y e_{j i}:\right. \\
& \left.\qquad v \in V, x \in(V \cap H) a_{i} \text {, and } y \in a_{j}^{-1} H\right\}
\end{aligned}
$$

are quadratic ideals of $J$. One should notice that $x e_{i i}=a^{-1} b e_{i i}, b \in V \cap H$, is in $J_{i i}$ as described above since $x=\left(a_{i}^{-1} b a_{i}^{-1}\right) a_{i}$ and $a_{i}^{-1} b a_{i}^{-1} \in H$. Also, if $A_{L}(T)=L$ and $A_{R}(L)=T$ then

$$
U_{\left[Q_{i j}^{\prime}(L)\right]_{i j}}\left(\left[Q_{j i}(T)\right]_{i j}\right)=0 .
$$

Is $Q_{i j}^{\prime}(L)$ the $i j$-annihilator of $Q_{j i}(T)$ ?

Lemma 3.2. Let $R$ be a prime associative ring and $W$ an additive subgroup of $(R,+)$. Then $A(W)=\{a \in R: a w R w a=0$ for all $w \in W\}=A_{L}(W) \cup A_{R}(W)$.

Proof. Let $a \in A(W)$ and suppose $w a \neq 0$ for some $w \in W$, so that $a w=0$. Suppose $a w^{\prime} \neq 0$ for some $w^{\prime} \in W$, but $a\left(w+w^{\prime}\right)=0$ or $\left(w+w^{\prime}\right) a=0$, both of which lead to a contradiction.

Thus in the *-prime case, we see that $A(W)=\{a \in R: a w R w a=0$ for all $w \in W\}$ where $W$ is an additive subgroup of $(R,+)$ is equal to $\bigcup_{i=1}^{4} K_{i}(W)$ where

$$
\begin{aligned}
& K_{1}(W)=\left\{a \in R: a W \subseteq P \text { and } a W \subseteq P^{*}\right\} \\
& K_{2}(W)=\left\{a \in R: a W \subseteq P \text { and } W a \subseteq P^{*}\right\} \\
& K_{3}(W)=\left\{a \in R: W a \subseteq P \text { and } W a \subseteq P^{*}\right\} \\
& K_{4}(W)=\left\{a \in R: W a \subseteq P \text { and } a W \subseteq P^{*}\right\}
\end{aligned}
$$

Lemma 3.3. Let $R$ be a $*$-prime associative ring and $J=H\left(R_{n}, \gamma_{a}\right)$. Let $B$ be a quadratic ideal of $J$ and $W$ be the set of elements of $R$ which occur as ji-components of elements of $B_{i j}$ (using the standard matrix units). A necessary and sufficient condition that $B$ bas a nonzero ij-annibilator is that the quadratic ideal $Q$ generated by $[A(W)]_{i j}=\left\{x_{i j}: x \in \bigcup K_{i}(W)\right\}$ is nonzero and $Q_{i j}=[A(W)]_{i j^{*}}$ Moreover, if this condition is satisfied then $Q=i j$-ann $(B)$.

Proof. By the definition of $i j$-annihilators, it suffices to show that $[A(W)]_{i j}=$ $\left\{x_{i j} \in J_{i j}: U_{x_{i j}}\left(B_{i j}\right)=0\right\}$.

Let $B$ and $W$ be as stated above so that $B_{i j}=\left\{w_{j i}: w \in W\right\}$.

Suppose $U_{x}\left(B_{i j}\right)=0$ and $x_{i j}=x e_{i j}+a_{j}^{-1} x^{*} a_{i} e_{j i}$ so that $0=U_{x_{i j}} U_{w_{j i}}\left(y_{i j}\right)=$ $x w y w x e_{i j}+a_{j}^{-1}(x w y w x)^{*} a_{i} e_{j i}$ for all $y \in R, w \in W$ and hence $x \in \bigcup K_{i}(W)^{i i}$.

Now suppose $x \in \bigcup K_{i}(W)$ and $x_{i j}=x e_{i j}+a_{j}^{-1} x^{*} a_{i} e_{j i} \quad U_{x_{i j}}\left(B_{i j}\right)=$ $\left\{x w x e_{i j}+a_{j}^{-1}(x w x)^{*} a_{i} e_{j i}: w \in W\right\}$. Since $x \in \bigcup K_{i}(W)$, one of the following is the case: 
$x w, w^{*} x^{*} \in P ; \quad x w, x^{*} w^{*} \in P ; \quad w x, x^{*} w^{*} \in P ; \quad w x, w^{*} x^{*} \in P$.

In any case, $x w x \in P \cap P^{*}=0$ so that $U_{x_{i j}}\left(B_{i j}\right)=0$.

Remark. Lemma 3.3 tells us that a necessary condition for $B$ to have a nonzero $i j$-annihilator is that $U K_{i}(W)$ be an additive subgroup of $(R,+)$, but in this case one can show that $U K_{i}(W)=K_{q}(W)$ for some $1 \leq q \leq 4$ by showing that if

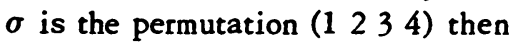

(a) $\cup K_{i}(W)=K_{j}(W) \cup K_{j \sigma}(W) \cup K_{j \sigma 2}(W)$, for one $j=1$ or 3 , and

(b) $K_{j}(W) \subseteq K_{j \sigma}(W)$ or $K_{j \sigma}(W) \subseteq K_{j}(W)$ for $1 \leq j \leq 4$.

The proof of this Remark is straightforward.

Lemma 3.4. Let $T$ be a nonzero right ideal of $R ; L=A_{L}(T)$; and $T^{\prime}=T P$ $+T P^{*}$. Then

(i) if $R$ is prime then $\left[i j \text {-ann }\left(Q_{j i}(T)\right)\right]_{i j}=\left[Q_{i j}^{\prime}(L)\right]_{i j}$ and

(ii) if $R$ is *-prime (not prime) then $\left[i j \text { ann }\left(Q_{j i}\left(T^{\prime}\right)\right)\right]_{i j}=\left[Q_{i j}^{\prime}(L)\right]_{i j} \cdot$

Proof. (i) Since $R$ is prime, the right annihilator of $T$ is zero. Thus $U K_{i}(T)$ $=K_{1}(T)=L$, and, by Lemma 3.3, $\left[Q_{i j}^{\prime}(L)\right]_{i j}=\left[i j \text {-ann }\left(Q_{j i}(T)\right)\right]_{i j}$

(ii) Since $R$ is *-prime (not prime), $P \neq 0$ so that $T^{\prime} \neq 0$.

We shall show $\bigcup K_{i}\left(T^{\prime}\right)=L$. Clearly $L \subseteq \bigcup K_{i}\left(T^{\prime}\right)$. Let $a \in K_{i}\left(T^{\prime}\right)$ so that $a T P a=0=a T P^{*} a$. aTPa $=0$ implies $a \in P^{*}$ or $a T \subseteq P^{*}$ and $a T P^{*} a=0 \mathrm{im}$ plies $a \in P$ or $a T \subseteq P$. Thus $a \in L$ and $U K_{i}\left(T^{\prime}\right)=L$, so that, by Lemma 3.3, $\left[i j \text {-ann }\left(Q_{j i}\left(T^{\prime}\right)\right)\right]_{i j}=\left[Q_{i j}^{\prime}(L)\right]_{i j}$

Theorem 3.5. If $J$ satisfies ACC on ij-annibilators and $R$ is a*-prime associative ring then $R$ satisfies ACC on left annibilator ideals.

Proof. In Lemma 3.4, we showed that if $L_{1} \subseteq L_{2} \subseteq L_{3} \subseteq \cdots$ is an ascending chain on left annihilator ideals then we may form an ascending chain, $B_{1} \subseteq B_{2} \subseteq$ $B_{3} \subseteq \cdots$ of $i j$-annihilators such that $\left(B_{k}\right)_{i j}=\left[Q_{i j}^{\prime}\left(L_{k}\right)\right]_{i j}$. Since the chain of $i j$-annihilators terminates and $B_{k}$ is generated by $\left(B_{k}\right)_{i j}$, we see that the chain of $\left[Q_{i j}^{\prime}\left(L_{k}\right)\right]_{i j}$ terminates so that the chain $L_{1} \subseteq L_{2} \subseteq L_{3} \subseteq \cdots$ terminates.

Summarizing results to this point we have:

Theorem 3.6. Let $J=H\left(R_{n}, \gamma_{a}\right), n \geq 2$, be a prime Jordan ring containing no infinite direct system of ij-quadratic ideals and satisfying ACC on ij-annibilators and let $R$ be an alternative ring with characteristic $\neq 2,1 / 2 \in R$, such that the set of symmetric elements, $H$, of $R$ is contained in the nucleus $N$ of $R$. Then $R$ is $a *$-prime associative Goldie ring or $n$ is either 2 or 3 and $R$ is a Cayley-Dickson ring.

By definition of a Cayley-Dickson ring, if $Z$ is the center of $R$ (i.e., $Z=$ $\{z \in N: z x=x z$ for all $x \in R\})$ and $Z^{\prime}$ is the field of quotients of $Z$, then 
$R^{\prime}=Z^{\prime} \otimes_{Z} R$ is a Cayley-Dickson algebra. E. Kleinfeld [6] has shown that if $R$ is a prime alternative (not associative) ring, then $N=Z$. If $R$ is a Cayley-Dickson ring with involution * such that $H \subseteq N$ then $H=Z$. This is due to the fact that, if we let $R^{\prime}$ be the Cayley-Dickson algebra associated with $R$ and extend * on $R$ to ${ }^{*}$ on $R^{\prime}$ by $\left(z^{-1} \otimes r\right)^{*}=z^{*-1} \otimes r^{*}$ for $z \in Z$ and $r \in R$, then $R^{\prime}$ is a CayleyDickson algebra with its symmetric elements invertible in its nucleus, so that * is a standard involution (see [5]). This gives us that $x^{*} x=x x^{*}$ for all $x \in R$, and that $H=Z=N$.

IV. If. We first consider the case when $R$ is a Cayley-Dickson ring. Let $R^{\prime}$ be the associated Cayley-Dickson algebra and consider $R$ as a subring of $R^{\prime}$ and * extended to $R^{\prime}$. We shall write $z^{-1} r$ for $z^{-1} \otimes r$. Thus $R^{\prime}=Z^{\prime} R$ where $Z^{\prime}$ is as above. If $J^{\prime}=H\left(R_{n}^{\prime}, \gamma_{a}\right)$ then $J^{\prime}$ is a $Z^{\prime}$ algebra and $J \subseteq J^{\prime}$.

Let $J=H\left(R_{n}, \gamma_{a}\right), n=2$ or 3 where $R$ is a Cayley-Dickson ring with $H \subseteq N$ and $1 / 2 \in R$. Let $b_{i j}$ and $x_{j i}$ be elements in $J_{i j}, i \neq j$, where $b_{i j}=b e_{i j}+$ $b^{*} a_{j}^{-1} a_{i} e_{j i}$ and $x_{j i}=x^{*} a_{j} a_{i}^{-1} e_{i j}+x e_{j i_{*}^{*}}$ Then since $a_{i}^{\prime}$ 's are in the center $Z(R)$, we have that $U_{b}\left(x_{j i}\right)=b x b e_{i j}+b^{*} x^{*} b^{*} a_{j}^{-1} a_{i} e_{j i}$. From this it is clear that $U_{b}\left(Z^{\prime} x_{j i}\right)$ is a one dimensional subspace of $J^{\prime}$ provided $b x b \neq 0$. But if $R^{\prime}=$ $Z_{R}^{\prime} R^{i j}$ then $b R^{\prime} b \neq 0$ for $b \neq 0$, so that $b R b \neq 0$ for $b \neq 0$. Thus $U_{b}\left(J_{i j}^{\prime}\right)$ is a subspace of $J^{\prime}$ of dimension greater than or equal to one for $b_{i j} \neq 0$.

Theorem 4.1. Let $J=H\left(R_{n}, \gamma_{a}\right), n=2$ or 3 , be a Jordan matrix ring. If $R$ is a Cayley-Dickson ring with involution such that $H \subseteq N$ then $J$ contains no infinite direct system of $i j-q u a d r a t i c$ ideals, $i \neq j$.

Proof. Suppose the theorem is false. That is, $J$ contains an infinite direct system of $i j$-quadratic ideals, $i \neq j$, say $\left\{Q_{k}\right\}$. Picking nonzero $q_{k} \in Q_{k}$ for each $k$, we obtain the system $\left\{U_{q_{k}}\left(J_{i j}^{\prime}\right)\right\}$ of nonzero $Z^{\prime}$ subspace of $J^{\prime}$. By the finite dimensionality of $J^{\prime}$ over $Z^{\prime}$, we see for some choice of $q_{k}$ and $q_{m}$ 's not equal to $q_{k}$ that

$$
U_{q_{k}}\left(J_{i j}^{\prime}\right) \cap\left[\sum_{m} U_{q_{m}}\left(J_{i j}^{\prime}\right)\right] \neq 0
$$

Thus

$$
0 \neq U_{q_{k}}\left(z^{-1} y_{i j}\right)=\sum_{m} U_{q_{m}}\left(z_{m}^{-1} y_{i j}^{(m)}\right)
$$

for some choice of $z, z_{m} \in Z$ and $y_{i j}, y_{i j}^{(m)} \in J_{i j}$. Therefore setting $\pi$ equal to the product of the $z_{m}$ 's we have

$$
0 \neq U_{q_{k}}\left(\pi y_{i j}\right)=\sum_{m}\left(U_{q_{m}}\left(\pi z_{m}^{-1} y_{i j}^{(m)}\right)\right) .
$$


However, $\pi z_{m}^{-1} y_{i j}^{(m)}$ and $\pi y_{i j}$ are elements in J. This contradicts the assumption that $\left\{Q_{k}\right\}$ is an infinite direct system.

Theorem 4.2. Let $J=H\left(R_{n}, \gamma_{a}\right), n=2$ or 3 , be a Jordan matrix ring. If $R$ is a Cayley-Dickson ring with involution such that $H \subseteq N$ then $J$ satisfies ACC on $i j$-annibilators, $i \neq j$.

Proof. Let $Q_{i}=i j$-ann $\left(B_{i}\right)$ and $0 \neq Q_{1} \subseteq Q_{2} \subseteq Q_{3} \subseteq \cdots$ be an ascending chain of $i j$-annihilators in J. Let $Q_{i}^{\prime}=Z^{\prime} Q_{i}$ The chain $Q_{1}^{\prime} \subseteq Q_{2}^{\prime} \subseteq Q_{3}^{\prime} \subseteq \ldots$ terminates since it is a chain of subspaces in a finite dimensional space. Thus there exists a $Q_{m}^{\prime}=\bigcup Q_{i}^{\prime}$.

Now, we show that $Q_{m}=\bigcup Q_{i}$. It suffices to show $Q_{m} \cap J_{i j}$ contains $\left(U Q_{i}\right)$ $\cap J_{i j}$ since $Q_{i}$ is generated by $Q_{i} \cap J_{i j^{*}}$ Let $x \in\left(\cup Q_{i}\right) \cap J_{i j}$ so that $x$ is in $Q_{m}^{\prime}$. That is, for some $s$ and $t$ in $Z$ and $y$ in $Q_{m} \cap J_{i j} x=s^{-1} t y$. Thus $U_{x}\left(B_{m} \cap J_{i j}\right)=U_{s^{-1} t}\left(B_{m} \cap J_{i j}\right)=\left(s^{-1} t\right)^{2} U_{y}\left(B_{m} \cap J_{i j}\right)=0$ and we see that $x$ is an element in $J_{i j}$ which annihilates $B_{m} \cap J_{i j}$ so that $x$ is in ijann $\left(B_{m}\right)=Q_{m}$.

The primeness of $J=H\left(R_{n}, \gamma_{a}\right), n=2$ or 3 , when $R$ is a Cayley-Dickson ring follows from the fact that $H\left(R_{n}^{\prime}, \gamma_{a}\right)=Z^{\prime} J$.

We now turn our attention to the case when $R$ is a *-prime associative Goldie ring. The primeness of $J$ follows from the involution primeness of $R_{n}$ under $\gamma_{a}$ [5, p. 129].

Until stated otherwise we will make the following assumption which will lead us to a contradiction. We shall assume that $J$ contains an infinite direct system, $\left\{Q_{k}\right\}$, of $i j$-quadratic ideals for some $i \neq j$, and $R$ is a *-prime Goldie ring.

Since $R$ is a *-prime Goldie ring with involution $R$ is both left and right Goldie so that $R / P$ and $R / P^{*}$ are each both left and right Goldie [4, p. 268]. Thus $R / P$ is a left and right order in the complete matrix ring, $D_{w}$, over a division ring $D$.

Let $\left\{f_{b b^{\prime}}\right\}$ be the standard set of matrix units in $D_{w}$. Every element in $D_{w}$ may be written as $\Sigma a_{b b^{\prime}} f_{b b^{\prime}}$ where $a_{b b^{\prime}}$ is an element in the centralizer of $\left\{f_{b b}\right\}$. We shall consider the coefficients, $a_{b b^{\prime}}$, to be lexicographically ordered according to their subscripts. We shall say that $\left(a_{b b^{0}}\right) \in D_{w}$ has $l$-zeros if the first $l$ coefficients are zero and if $l \neq w^{2}$ then the $(l+1)$ st one is not zero.

We shall consider $R$ as being a subring of the direct sum $R / P+R / P^{*}$. Let $g$ and $g_{*}$ be the projections

$$
g: R / P+R / P^{*} \rightarrow R / P, \quad g_{*}: R / P+R / P^{*} \rightarrow R / P^{*} .
$$

Also let

$$
M_{i j}\left(Q_{k}\right)=\left\{g(x): x \in R \text { and } x_{i j} \in Q_{k}\right\} \text { and } M_{i j}^{*}\left(Q_{k}\right)=\left\{g_{*}(x): x \in R \text { and } x_{i j} \in Q_{k}\right\} \text {. }
$$

Since $R / P$ and $R / P^{*}$ are prime Goldie rings we may consider each as a subring 
of a matrix ring over a division ring, so that $M_{i j}\left(Q_{k}\right)$ and $M_{i j}^{*}\left(Q_{k}\right)$ are sets of matrices and it makes sense to talk about the zeros of their elements. It should be pointed out that in light of this situation the elements of $J$ are matrices whose entries are ordered pairs of matrices since $R \subseteq R / P+R / P^{*}$.

Lemma 4.3. If each $Q_{k} \cap J_{i j}$ contains a nonzero element $x^{(k)}=x_{k} a_{j} e_{i j}+$ $x_{k}^{*} a_{i} e_{j i}$ such that $g\left(x_{k} a_{j}\right)$ bas l-zeros, $l \neq w^{2}$ where viewed as a matrix in $R / P$, then $J$ contains an infinite direct system of ij-quadratic ideals $\left\{A_{p}\right\}$ such that each $A_{p} \cap J_{i j}$ contains a nonzero element $y^{(p)}=y_{p} a_{j} e_{i j}+y_{p}^{*} a_{i} e_{j i}$ such that

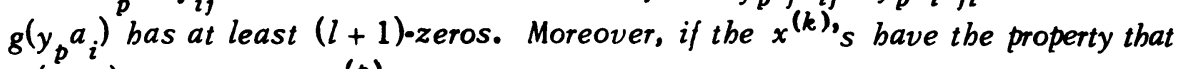

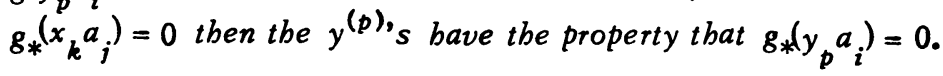

Proof. If we let $A_{p}$ be the quadratic ideal generated by $Q_{p}+Q_{p+1}$ for odd integers then $\left\{A_{p}\right\}$ is an infinite direct system of $i j$-quadratic ideals of $J$. We shall use the existence of $x^{(p)}$ and $x^{(p+1)}$ to construct $y^{(p)}$ in $A_{p}$.

Let $p$ be an odd integer and let $x^{(p)}$ and $x^{(p+1)}$ be as in the statement of the lemma. Suppose the $(l+1)$ st position is the one corresponding to the pair $(r, s)$ so that

$$
g\left(x_{p} a_{j}\right)=\left(a_{b b^{\prime}}\right)=\left\{\begin{array}{l}
0 \text { if } b<r \text { or if } b=r \text { but } b^{\prime}<s \\
a_{r s} \neq 0 \\
? \quad \text { otherwise, }
\end{array}\right.
$$

and

$$
g\left(x_{p+1} a_{j}\right)=\left(b_{b b^{\prime}}\right)=\left\{\begin{array}{l}
0 \text { if } b<r \text { or if } b=r \text { but } b^{\prime}<s \\
b_{r s} \neq 0, \\
? \text { otherwise. }
\end{array}\right.
$$

By the Faith-Utumi theorem, the centralizer $D$ of $\left\{f_{b b^{\prime}}\right\}$ contains a left and right order $I$ such that $\Sigma I f_{b b^{\prime}} \subseteq R / P$. Thus every element in $D$ may be written as $c^{-1} d=u v^{-1}$ for some $c, \bar{d}, u, v \in I$ so that $a_{r s}=c^{-1} d=u v^{-1}$ for $c, d, u, v$ in $I$ and $b_{r s}=c_{0}^{-1} d_{0}=u_{0} v_{0}^{-1}$ for $c_{0}, d_{0}, u_{0}, v_{0}$ in $I$. Since $I$ is a left and right order in $D$, by Ore's theorem [4, p. 262] there exist nonzero elements $x, x_{0}, y, y_{0}$ in $I$ such that $x d=x_{0} d_{0}$ and $u y=u_{0} y_{0}$. Now, let $m$ and $q$ be elements of $R$ such that

$$
g(m)=\left(m_{b b^{\prime}}\right)= \begin{cases}0 & \text { if } b \neq s \text { or } b^{\prime} \neq r, \\ v y x c & \text { if } b=s \text { and } b^{\prime}=r\end{cases}
$$

and

$$
g(q)=\left(q_{b b^{\prime}}\right)= \begin{cases}0 & \text { if } b \neq s \text { or } b^{\prime} \neq r, \\ v_{0} y_{0} x_{0} c_{0} & \text { if } b=s \text { and } b^{\prime}=r .\end{cases}
$$

Since $g(R)=R / P$, such an $m$ and $q$ exist in $R$. 
Let $m_{j i}=a_{i}^{-1} m^{*} a_{j} e_{i j}+m e_{j i}$ and $q_{j i}=a_{i}^{-1} q^{*} a_{j} e_{i j}+q e_{j i}$. Here $e_{i j}$ and $e_{j i}$ are elements in the set of matrix units in $R_{n}$. Thus

$$
\begin{aligned}
U_{x}^{(p)}\left(m_{j i}\right) & =\left(x_{p} a_{j} e_{i j}+x_{p}^{*} a_{i} e_{j i}\right)\left(a_{i}^{-1} m^{*} a_{j} e_{i j}+m e_{j i}\right)\left(x_{p} a_{j} e_{i j}+x_{p}^{*} a_{i} e_{j i}\right) \\
& =\left(x_{p} a_{j}\right) m\left(x_{p} a_{j}\right) e_{i j}+x_{p}^{*} m^{*} a_{j} x_{p}^{*} a_{i} e_{j i} .
\end{aligned}
$$

But

$$
\left(x_{p} a_{j}\right) m\left(x_{p} a_{j}\right)=g\left(x_{p} a_{j}\right) g(m) g\left(x_{p} a_{j}\right)+g_{*}\left(x_{p} a_{j}\right) g_{*}(m) g_{*}\left(x_{p} a_{j}\right)
$$

and

$$
\begin{aligned}
g\left(x_{p} a_{j}\right) g(m) g\left(x_{p} a_{j}\right) & =\left(a_{b b^{\prime}}\right)\left(m_{b b^{\prime}}\right)\left(a_{b b^{\prime}}\right)=\left(a_{b b^{\prime}}\right)\left(\sum_{r} m_{s r} a_{r b^{\prime}}\right)_{s b^{\prime}} \\
& =\left(\sum_{s} a_{b s}\left(\sum_{r} m_{s r^{\prime}} a_{r b^{\prime}}\right)\right)_{b b^{\prime}}=\left(\sum_{s} \sum_{r} a_{b s^{m}} m_{s r^{\prime}} a_{r b^{\prime}}\right)_{b b^{\prime}} \\
& = \begin{cases}0 & \text { if } b<r \text { or if } b=r \text { but } b^{\prime}<s \\
u y x d & \text { if } b=r \text { and } b^{\prime}=s \\
? & \text { otherwise. }\end{cases}
\end{aligned}
$$

Similarly

$$
U_{x^{(p+1)}}\left(q_{j i}\right)=\left(x_{p+1} a_{j}\right) q\left(x_{p+1} a_{j}\right) e_{i j}+x_{p+1}^{*} q^{*} a_{j} x_{p+1}^{*} a_{i} e_{j i}
$$

where

$$
\left(x_{p+1} a_{j}\right) q\left(x_{p+1} a_{j}\right)=g\left(x_{p+1} a_{j}\right) g(q) g\left(x_{p+1} a_{j}\right)+g_{*}\left(x_{p+1} a_{j}\right) g_{*}(q) g_{*}\left(x_{p+1} a_{j}\right)
$$

and

$$
g\left(x_{p+1} a_{j}\right) g(q) g\left(x_{p+1} a_{j}\right)= \begin{cases}0 & \text { if } b<r \text { or } b=r \text { and } b^{\prime}<s, \\ u_{0} y_{0} x_{0} d_{0} & \text { if } b=r \text { and } b^{\prime}=s, \\ ? & \text { otherwise. }\end{cases}
$$

$U_{x}(p)\left(m_{j i}\right)$ is a nonzero element in $Q_{p}$ and $U_{x}(p+1)\left(q_{j i}\right)$ is a nonzero element in $Q_{p+1}^{x}$ so that $U_{x}(p)\left(m_{j i}\right)$ minus $U_{x}(p+1)\left(q_{j i}\right)$ is not equal to zero since $Q_{p} \cap Q_{p+1}$ $=0$. $g\left(x_{p} a_{j}\right) g(m) g\left(x_{p} a_{j}\right)-g\left(x_{p+1} a_{j}\right) g(q) g\left(x_{p+1} a_{j}\right)$ is an element of $D_{w}$ with at least $(l+1)$-zeros, since uyxd $=u_{0} y_{0} x_{0} d_{0}$. Let

$$
y^{(p)}=U_{x}^{(p)}\left(m_{j i}\right)-U_{x}^{(p+1)}\left(q_{j i}\right)
$$

$y^{(p)}$ is a nonzero element in $A_{p} \cap J_{i j}$ such that $g\left(y_{p} a_{j}\right)$ has at least $(l+1)$ zeros, where $y_{p}$ is taken to be $x_{p} a_{j} m x_{p}-x_{p+1} a_{j} q x_{p+1}$ so that $y^{(p)}=y_{p} a_{j} e_{i j}+$ $y_{p}^{*} a_{j} e_{j i}$

This completes the proof of the lemma, since the last statement of the lemma is clear from the construction. 
We use Lemma 4.3 to show that there is a contradiction built in the assumption stated above.

Theorem 4.4. Let $J=H\left(R_{n}, \gamma_{a}\right)$ where $R$ is associative and $n \geq 2$. If $R$ is *-prime Goldie then $J$ does not contain an infinite direct system of ij-quadratic ideals for all $i \neq j$.

Proof. Suppose that the theorem is false. We may assume $J$ contains an infinite direct system $\left\{Q_{k}\right\}$ such that each $Q_{k}$ contains an $x^{(k)}=x_{k} a_{j} e_{i j}+x_{k}^{*} a_{i} e_{j i}$ such that $g\left(x_{k} a_{j}\right)$ has $l$-zeros, $l \neq w^{2}$, for all integral values of $k$, since this or the corresponding statement using $g_{*}\left(x_{k} a_{j}\right)$ is true for some infinite subset of $\left\{Q_{k}\right\}$.

By Lemma 4.3, $J$ contains an infinite direct system $\left\{Q_{k}^{(1)}\right\}$ such that each $Q_{k}^{(1)} \cap J_{i j}$ contains a nonzero element $y^{(k)}=y_{k} a_{j} e_{i j}+y_{k}^{*} a_{i} e_{j i}$ such that $g\left(y_{k} a_{j}\right)$ has $m$-zeros where $m>l$.

Continuing by induction and Lemma 4.3, $J$ contains an infinite direct system $\left\{Q_{k}^{(r)}\right\}, r \leq w^{2}$, such that $Q_{k}^{(r)} \cap J_{i j}$ contains an element $c^{(k)}=c_{k} a_{j} e_{i j}+c_{k}^{*} a_{i} e_{j i}$ $\neq 0$ such that $g\left(c_{k} a_{j}\right)=0$. But since $c^{(k)} \neq 0$, it must be the case that $g_{*}\left(c_{k} a_{j}{ }_{j}\right)$ $\neq 0$.

Similar to what we did above, we may assume that $g_{*}\left(c_{k} a_{j}\right)$ has $m$-zeros for each $k$. Then we may go through an argument similar to the one just completed to obtain an infinite direct system $\left\{Q_{k}^{(r)}\right\}, r \leq 2 w^{2}$ such that $Q_{k}^{(r)} \cap J_{i j}$ contains an element $d^{(k)}=d_{k} a_{j} e_{i j}+d_{k}^{*} a_{i} e_{i j} \neq 0$ such that $g_{*}\left(d_{k} a_{j}\right)=0$. But the last sentence of Lemma 4.3 tells us that this construction may be done so that $g\left(d_{k} a_{j}\right)=0$. This is impossible.

We now show that $R$ *-prime Goldie implies that $J$ satisfies ACC on $i j$-annihilators.

Lemma 4.5. If $K_{q}\left(W_{1}\right) \subseteq K_{q}\left(W_{2}\right) \subseteq \cdots \subseteq K_{q}\left(W_{m}\right) \subseteq \cdots$ while $q=1,2,3,4$ and the $W_{m}$ 's are additive subgroups of $(R,+)$, then $K_{q}\left(W_{i}\right)=K_{q}\left(\Sigma_{j \geq i} W_{j}\right)$. (The notation used bere is that which was introduced in the proof of Lemma 3.2.)

Proof. If $q=1$ or 3 then we are talking about left or right annihilators in $R$, and hence the lemma is true for $q=1$ or 3 . Also, a slight adaptation of the following proof yields a proof for these cases.

Suppose $q=2$. Let $a$ be an element in $K_{2}\left(W_{i}\right)$ so that $a W_{j} \subseteq P$ and $W_{j} \subseteq \subseteq P^{*}$ for all $j \geq i$, since $K_{2}\left(W_{i}\right) \subseteq K_{2}\left(W_{j}\right)$ for $j \geq i$. Thus $a\left(\Sigma_{j \geq i} W_{j}\right) \subseteq \bar{P}$ and $\left(\Sigma_{j \geq i} W_{j}\right) a$ $\subseteq P^{*}$ so that $a$ is an element in $K_{2}\left(\Sigma_{j \geq i} W_{j}\right)$. Therefore $K_{2}\left(W_{i}\right)$ is contained in $K_{2}\left(\Sigma_{j \geq i} W_{j}\right)$. Clearly if $a$ is an element in $K^{2}\left(\Sigma_{j \geq i} W_{j}\right)$ then $a$ is an element in $K_{2}\left(W_{i}\right)$ since $a W_{i} \subseteq a\left(\Sigma_{j \geq i} W_{j}\right) \subseteq P$ and $W_{i} a \subseteq\left(\Sigma_{j \geq i} W_{j}\right) a \subseteq P$ and $W_{i} a \subseteq\left(\Sigma_{j \geq i} W_{j}\right) a \subseteq$ $P^{*}$. Thus $K_{2}\left(W_{i}\right)=K_{2}\left(\sum_{j \geq i} W_{j}\right)$.

The proof for $q=4$ is similar and therefore it is omitted. 
Theorem 4.6. Let $J=H\left(R_{n}, \gamma_{a}\right), n \geq 2$, where $R$ is associative and *-prime. If $R$ is Goldie then $J$ satisfies ACC on ij-annibilators.

Proof. From the Remark of $\$$ III, it suffices to show $R$ satisfies ACC on sets of the form $U K_{i}(T)$ where $T$ is an additive subgroup of $(R,+)$. Thus it suffices to show $R$ satisfies ACC on sets of the form $K_{q}(W)$ where $W$ is an additive subgroup of $(R,+)$.

Suppose we have such a chain $K_{q}\left(W_{1}\right) \subseteq K_{q}\left(W_{2}\right) \subseteq \ldots \subseteq K_{q}\left(W_{m}\right) \subseteq \ldots$ By Lemma 4.5, we may assume that $w_{1} \supseteq w_{2} \supseteq \cdots \supseteq w_{m} \supseteq \cdots$.

By ACC on left and right annihilators in $R / P$, we have the following chains in $R / P$ terminating

$$
A_{R}\left(W_{i} / P\right) \subseteq A_{R}\left(W_{i+1} / P\right), \quad A_{L}\left(W_{i} / P\right) \subseteq A_{L}\left(W_{i+1} / P\right)
$$

and correspondingly in $R / P^{*}$

$$
A_{R}\left(W_{i} / P^{*}\right) \subseteq A_{R}\left(W_{i+1} / P^{*}\right), \quad A_{L}\left(W_{i} / P^{*}\right) \subseteq A_{L}\left(W_{i+1} / P^{*}\right) .
$$

Hence the following chains terminate in $R$ :

$\left\{a \in R: a+P \in A_{R}\left(W_{i} / P\right)\right\} \subseteq\left\{a \in R: a+P \in A_{R}\left(W_{i+1} / P\right)\right\}$,

$\left\{a \in R: a+P \in A_{L}\left(W_{i} / P\right)\right\} \subseteq\left\{a \in R: a+P \in A_{L}\left(W_{i+1} / P\right)\right\}$,

$\left\{a \in R: a+P^{*} \in A_{R}\left(W_{i} / P^{*}\right)\right\} \subseteq\left\{a \in R: a+P^{*} \in A_{R}\left(W_{i+1} / P^{*}\right)\right\}$,

$\left\{a \in R: a+P^{*} \in A_{L}\left(W_{i} / P^{*}\right)\right\} \subseteq\left\{a \in R: a+P^{*} \in A_{L}\left(W_{i+1} / P^{*}\right)\right\}$,

But the chain $K_{q}\left(W_{i}\right) \subseteq K_{q}\left(W_{i+1}\right)$ is the intersection of corresponding terms of two chains which terminate. Hence we have that the chain $K_{q}\left(W_{i}\right) \subseteq K_{q}\left(W_{i+1}\right)$ terminates.

V. Quotients. In order to complete the proof of the Main Theorem, the last statement is all that needs to be shown.

In the case when $R$ is a *-prime Goldie ring this follows from [2].

Let $R$ be a Cayley-Dickson ring and let $R^{\prime}$ be the Cayley-Dickson algebra associated with $R$. Extend the involution on $R$ to the standard involution on $R^{\prime}$ and extend $\gamma_{a}$ on $R_{n}$ to $\gamma_{a}$ on $R^{\prime}$. We need to show that every element in $J^{\prime}=$ $H\left(R_{n}^{\prime}, \gamma_{a}\right), n=2,3$, has the form $U_{a}^{-1}(b)$ for some $a$ and $b$ in $J$ with $a$ regular in. $J$, and that regular elements in $J$ are invertible in $J^{\prime}$.

Since every element in $R^{\prime}$ is of the form $z^{-1} b$ for $0 \neq z$ in the center $Z=$ $Z(R)$ and $b$ in $R$, given an element $t$ in $H\left(R_{n}^{\prime}, \gamma_{a}\right)$ we may express it as an $n \times n$ matrix $\left(c_{k k^{\prime}}\right)$ where each entry $c_{k k^{\prime}}$ is of the form $z_{k k^{\prime}}^{-1} b_{k k^{\prime}}$ for $z_{k k^{\prime}} \neq 0$ in $Z(R)$ $=H(R)$ and $b_{k k^{\prime}}$ in $R$. We shall exhibit a $y$ and a $w$ such that $t=U_{w}^{-1}(y)$. Let $\pi$ be the product of $z_{k k}{ }^{\prime}$ 's so that $\pi^{2} z_{k k}^{-1}$ ' is in $H(R)$ and let $w$ be the diagonal 
matrix $\pi e_{11}+\cdots+\pi e_{n n^{*}}$ Let $y=\left(\pi^{2} z_{k k}^{-1}, b_{k k}\right)$. Then $y$ is in $H\left(R_{n}, \gamma_{a}\right)$ since

$$
\gamma_{a}(y)=\gamma_{a}\left(\pi^{2} z_{k k^{\prime}}, b_{k k^{\prime}}\right)=\pi^{2} \gamma_{a}\left(z_{k k^{-1}}, b_{k k^{\prime}}\right)=\pi^{2}\left(z_{k k^{-1}}, b_{k k^{\prime}}\right)=\left(\pi^{2} z_{k k^{-1}}, b_{k k^{\prime}}\right)=y \text {. }
$$

Clearly $y$ and $w$ have the desired property.

Finally, let $x$ be a regular element of $J$. Let $t$ be an arbitrary element in $J^{\prime}$. By what was shown above, $t=U_{w^{-1}}(y)$ for $w$ in $Z(R)$ and $y$ in $J$. Here we are considering $J^{\prime}$ as a $Z^{\prime}$-algebra where $Z^{\prime}$ is the field of quotients for $Z(R)$ and identifying $z^{-1}$ with $z^{-1} 1$ for 1 in $J^{\prime}$. Hence

$$
U_{x}(t)=U_{x} U_{w^{-1}}(y)=U_{x}\left(w^{-2} y\right)=w^{-2} U_{x}(y) \neq 0
$$

since $x$ is regular in $J$. Thus $U_{x}$ is $1-1$ on the finite dimensional $Z^{\prime}$-algebra $J^{\prime}$. Recall $R^{\prime}$ is 8-dimensional over $Z^{\prime}$. Therefore, $U_{x}$ is a $1-1$ linear transformation on a finite dimensional vector space and hence $U_{x}$ is onto so that 1 is in $U_{x}\left(J^{\prime}\right)$ and $x$ is invertible.

\section{BIBLIOGRAPHY}

1. D. J. Britten, On Cayley-Diekson rings, Canad. Math. Bull. Math. Notes (to appear). 2. - On prime Jordan rings $H(R)$ with chain condition, J. Algebra (to appear).

3. T. S. Erickson and S. Montgomery, The prime radical in special Jordan rings, Trans. Amer. Math. Soc. 156 (1971), 155-164. MR 43 \#306.

4. N. J acobson, Structure of rings, 2nd. ed., Amer. Math. Soc. Colloq. Publ., vol. 37, Amer. Math. Soc., Providence, R. I., 1964. MR 36 \#5158.

5. —_, Structure and representations of Jordan algebras, Amer. Math. Soc. Colloq. Publ., vol. 39, Amer. Math. Soc., Providence, R. I., 1968. MR 40 \#4330.

6. E. Kleinfeld, Primitive alternative rings and semisimplicity, Amer. J. Math. 77 (1955), 725-730. MR 17, 231.

7. J. M. Osbom, Varieties of algebras, Advances in Math. 8 (1972), 163-369. MR 44 \#6775.

8. C. Tsai, The prime radical in a Jordan ring, Proc. Amer. Math. Soc. 19 (1969), 1171-1175. MR 37 \#6336.

DEPAR TMENT OF MATHEMATICS, UNIVERSITY OF IINDSOR, WINDSOR, ONTARIO, N9B 3P4, CANADA 